\title{
The infancy of supernova remnants: evolving a supernova into its remnant in $3 \mathrm{D}$
}

\author{
Michael Gabler ${ }^{1}$, Hans-Thomas Janka ${ }^{1}$ and Annop Wongwathanarat ${ }^{2}$ \\ ${ }^{1}$ Max-Planck-Institut für Astrophysik, Karl-Schwarzschild-Str. 1, 85741 Garching, Germany \\ ${ }^{2}$ RIKEN, Astrophysical Big Bang Laboratory, 2-1 Hirosawa, Wako, Saitama 351-0198, Japan
}

\begin{abstract}
Recently, first neutrino-driven supernova explosions have been obtained in 3D, selfconsistent, first-principle simulations, these models are still not always exploding robustly and, in general, the explosions are not sufficiently energetic. To constrain the explosion mechanism, and the related uncertainties, it is thus very helpful to consider observational constraints: pulsar kicks, progenitor association and supernova remnants (SNR). Recent observations of asymmetries in the supernova ejecta of Cas A are very promising, to compare to long-term simulations of the explosion. In addition 3D observations of SN87A are becoming more constraining on the geometry of the ejected material during the explosion. In this talk I will discuss our efforts to model the late time evolution of a 3D supernova explosion, where we include the effects of beta decay, which inflates the structures rich in ${ }^{56} \mathrm{Ni}$. The structures we find in the simulations depend on the quantities plotted.
\end{abstract}

Keywords. hydrodynamics, instabilities, methods: numerical, (stars:) supernovae: individual (1987A), (stars:) supernovae: general, (ISM:) supernova remnants

\section{Introduction}

Supernovae are amongst the most energetic events in our universe. Huge efforts have been undertaken to improve our knowledge about these phenomena by performing sophisticated simulations and by ever more detailed observations. Our current understanding of, in particular, core-collapse supernovae (CCSNe) shows us that 3D effects are very important to model them theoretically, but also to interpret the observations. First long-term multi-dimensional supernova simulations became possible at end of the 1980s and in the early 1990s (Arnett et al. (1989), Fryxell et al. (1991), Mueller et al. (1991), Hachisu et al. (1990), Hachisu et al. (1992), Hachisu et al. (1994), Yamada \& Sato (1990), Herant \& Benz (1991), Herant \& Benz (1992), Herant \& Woosley (1994), Iwamoto et al. (1997), Nagataki et al. (1998)). Later the complexity of the simulations was increased, by among other things no longer initiating the explosions with thermal bombs (Kifonidis et al. (2000), Kifonidis et al. (2003), Hungerford et al. (2003), Hungerford et al. (2005), Kifonidis et al. (2006), Joggerst et al. (2009), Joggerst et al. (2010), Joggerst et al. (2010), Couch et al. (2009), Gawryszczak et al. (2010), Couch et al. (2011), Ono et al. (2013), Ellinger et al. (2012), Ellinger et al. (2013)). In a series of papers, we also investigated how neutrino-driven explosions evolve through the progenitor up to its surface and how multi-dimensional effects and instabilities create asymmetries in the ejecta (Hammer et al. (2010), Wongwathanarat et al. (2010), Wongwathanarat et al. (2013), Wongwathanarat et al. (2015), Wongwathanarat et al. (2016)).

Other groups followed a different approach to investigate the CCSN phenomenon. They modeled particular supernova remnants such as SN 1987A or Cas A (Li et al. (1993), Blondin \& Lundqvist (1993), Blondin et al. (2001), Tanaka \& Washimi (2002), 
Podsiadlowski et al. (2007), Dewey et al. (2012), Potter et al. (2014), Orlando et al. (2015), Orlando et al. (2016)). These studies focused on the late phases of the explosion/early phases of the remnant and the interaction with the circumstellar material. The simulations were started with spherically symmetric or artificial initial conditions after the shock break-out.

The theoretical models were stimulated by the ever more detailed observations revealing truly 3D structures and asymmetries (Nagataki et al. (1998), Fesen (2001), Hwang et al. (2004), Fesen et al. (2006), Milisavljevic \& Fesen (2013), Grefenstette et al. (2014), Milisavljevic \& Fesen (2015), Boggs et al. (2015), Grefenstette et al. (2017)).

In the current work we continue our previous study (Wongwathanarat et al. (2015)) and simulate the evolution from a 3D supernova explosion starting at bounce up to the early remnant stage including nuclear beta decay of ${ }^{56} \mathrm{Ni}$ consistently.

\section{Numerics}

For our computations we use the 3D, explicit finite-volume hydrodynamic code PROMETHEUS (Fryxell et al. (1991), Mueller et al. (1991)). It is based on the piecewise parabolic reconstruction method (PPM; Colella \& Woodward (1984)), employs a Riemann solver for realistic gases (Colella \& Glaz (1985)) and treats multi-fluid systems with the consistent multi-fluid advection scheme (CMA; Plewa \& Müller (1999)). To avoid artefacts at the polar axis of a spherical polar grid and to allow for larger time steps due to a less restrictive CFL condition close to the polar axis, we use a 'YinYang' grid (Kageyama \& Sato (2004), Wongwathanarat et al. (2010)). The code has already been applied to calculate the 3D propagation of the shock and the ejecta during a neutrino-driven supernova explosion up to the shock break out (Wongwathanarat et al. (2013), Wongwathanarat et al. (2015)) and to investigate light curves for different progenitors compared to SN 1987A (Utrobin et al. (2015)) as well as to study the production of ${ }^{44} \mathrm{Ti}$ and ${ }^{56} \mathrm{Ni}$ in Cas A (Wongwathanarat et al. (2016)).

Here we extend the code version PROMETHEUS-HOTB by including the effects of additional heating due to beta decay. ${ }^{56} \mathrm{Ni}$ decays with a half-life time of $\tau_{1 / 2}^{N i}=6.07 \mathrm{~d}$ to ${ }^{56} \mathrm{Co}$, which in turn decays to the stable ${ }^{56} \mathrm{Fe}$ with $\tau_{1 / 2}^{C o}=77.23 \mathrm{~d}$. The energy releases in $\gamma$ photons and $\mathrm{e}^{+}$per decay are $Q_{N i}=1.72 \mathrm{MeV}$ and $Q_{C o}=3.735 \mathrm{MeV}$, respectively (Junde et al. (2011)). For the current study we assume that the total energy is deposited locally. This overestimates the energy trapped in the medium, because depending on the model, the ejecta become transparent to the photons released during the beta decay after about $100-150 \mathrm{~d}$. However, until this time about $2 / 3$ of the radioactive material will have decayed and our approximation only mildly overestimates the energy input due to radioactive decay. Practically, the additional energy is implemented as a source term for the specific energy per unit mass $\varepsilon$ and the energy increase in a time interval $\Delta t$ is given by

$$
\Delta \varepsilon=\sum_{i} \frac{Q_{i} X_{i}}{m_{i}}\left(1-e^{-\frac{\Delta t \ln 2}{\tau_{1 / 2}^{i}}}\right),
$$

where $X_{i}$ and $m_{i}$ are the mass fraction and atomic mass of the respective element $i=$ $\{N i, C o\}$. To reduce numerical costs and numerical diffusion we use a radially moving mesh with $v_{\text {grid }} \sim v_{r}$, which results in a quasi-Lagrangian grid, because $v_{r}>>\left\{v_{\theta}, v_{\varphi}\right\}$. 
model model in type $\operatorname{mass}\left[M_{\odot}\right] \operatorname{radius}\left[10^{6} \mathrm{~km}\right] \mathrm{t}_{\mathrm{map}}[\mathrm{ks}] \mathrm{t}_{\text {outbreak }}[\mathrm{ks}]$ Wongwathanarat +2015

\begin{tabular}{lllllll}
\hline W15 & W15-2-cw & RSG & 15 & 339 & 5.8 & 85 \\
L15 & L15-1-cw & RSG & 15 & 434 & 5.0 & 96 \\
N20 & N20-4-cw & BSG & 20 & 33.8 & 1.4 & 5.6 \\
B15 & B15-1-pw & BSG & 15 & 39.0 & 3.2 & 7.3
\end{tabular}

Table 1. Progenitor models used in this work.

\section{Models}

Continuing the work of Wongwathanarat et al. (2015) we consider two red (RSG) and two blue super giants (BSG) progenitors (see Table 1) and evolve them for up to two years including the beta decay of ${ }^{56} \mathrm{Ni}$ to ${ }^{56} \mathrm{Fe}$.

The evolution of the models until shock breakout in Wongwathanarat et al. (2015) can be summarized as follows: Initial asymmetries arise due to hydrodynamical instabilities behind the stalled shock during the first second of post bounce evolution. Once the shock expands the initial asymmetric structures move outwards and fragment due to RayleighTaylor (RT) instabilities at the $\mathrm{C}+\mathrm{O} / \mathrm{He}$ and $\mathrm{He} / \mathrm{H}$ shell interfaces of the progenitor. How strong the initial, big plumes fragment depends sensitively on the structure of the progenitor. In particular, the density decrease at each interface and the flatness of the density profiles within the following shell determine how much time the instabilities have to grow before the reverse shock hits the inner ejecta. At this point the plumes are decelerated and get compressed. Fragmentation and mixing occurs by the growth of RT instabilities in the region between reverse shock and shell interfaces. It is therefore suppressed when the reverse shock from the $\mathrm{He} / \mathrm{H}$ interface decelerates the inner ejecta before they reach the RT unstable region at the base of the $\mathrm{H}$ envelope. Depending on the progenitor structure, the different models have different characteristics at shock breakout. The two RSG (W15 and L15) are qualitatively similar: there are small scale RT-fingers grouped into larger structures that arose from the initial asymmetries during the shock revival phase. In the BSG N20 the initial asymmetries were decelerated and compressed due to a faster interaction with the reverse shock, before the inner ejecta could reach the RT unstable region at the $\mathrm{He} / \mathrm{H}$ interface. Thus, the model is quite spherical even at late times. Model B15 has different morphology than the other two groups. There are a few very elongated fingers in the direction of the initial asymmetries. Those almost did not interact with the reverse shock. However, the latter slowed down and compressed the material in the central region such that the fast moving fingers are much more extended than in the other models. For a more detailed description we refer to Wongwathanarat et al. (2015).

\section{Results}

Right after the shock breakout, the Ni-rich ejecta of almost all models still have not reached the homologous expansion. For a short time, the velocity differences of different structures lead to a further elongation of the Ni-fingers for models W15, L15 and in particular B15. The Ni-rich ejecta of the more spherical model N20 become slightly asymmetric. Starting at about one day, the radioactive decay of ${ }^{56} \mathrm{Ni}$ provides a significant energy source that heats up the Ni-clumps. This increase of the internal energy leads to an inflation of the corresponding structures. Small fragmented fingers start to expand and can even merge with neighbouring fingers. Thus, they create larger structures in the direction of the initial asymmetries from the onset of the explosion. In Figure 1, we plot the surfaces where the mass fraction of ${ }^{56} \mathrm{Ni}+{ }^{56} \mathrm{Co}+{ }^{56} \mathrm{Fe}$ is larger than $3 \%$. These 

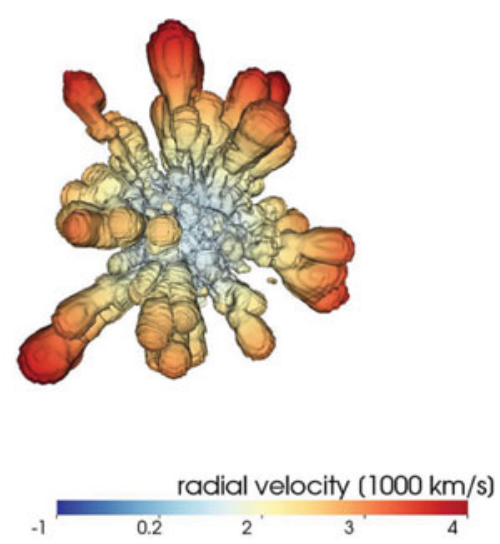

L15
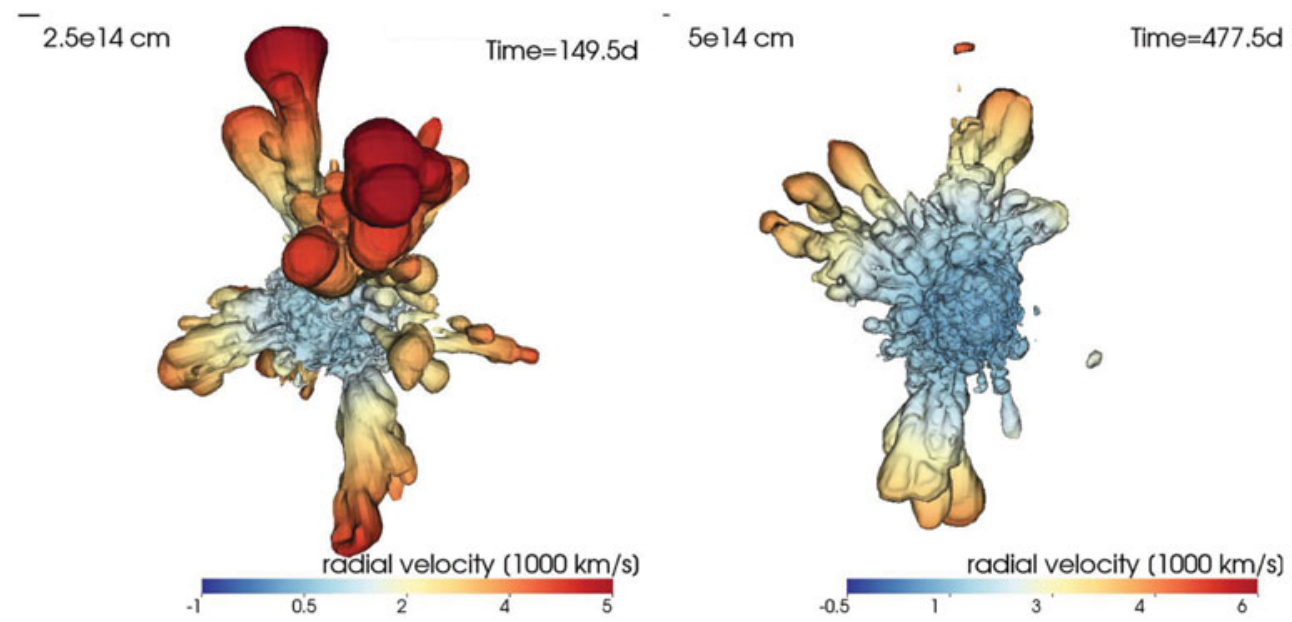
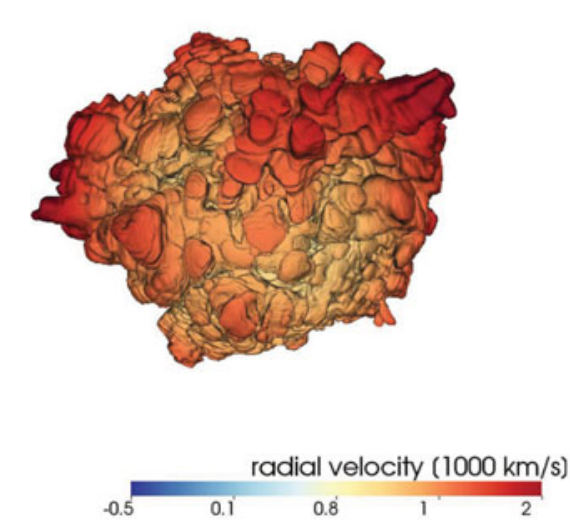

W15

Figure 1. Isosurfaces where the elements of the decay chain of ${ }^{56} \mathrm{Ni}$ sum up to $3 \%$. The color coding is the radial velocity.

surfaces contain most of the final ${ }^{56} \mathrm{Fe}$ and are thus representative for the most extended structures of the ejecta.

At late times, the small asymmetries in model N20 after the shock breakout, are almost completely washed out and the overall shape of the ejecta is rather close to spherical symmetry. In the other models the reverse shock slows down most of the central material which therefore forms an almost spherical shell (blue regions in panels for B15, L15, and W15 in Figure 1), while the fingers become more elongated and expand with modest extra boost by the ${ }^{56} \mathrm{Ni}$ decay heating. In the models L15 and W15 we see three main structures consisting of a few Ni-fingers. In addition, L15 has some smaller structures with only one or a few smaller fingers. Model B15 has more fingers than the two RSG, but the basic morphology elements are highly asymmetric and comparable in all three models.

Due to the expansion of the Ni-bubbles or Ni-fingers their density decreases with respect to the surrounding and matter gets accumulated at the walls of the bubbles/fingers. 


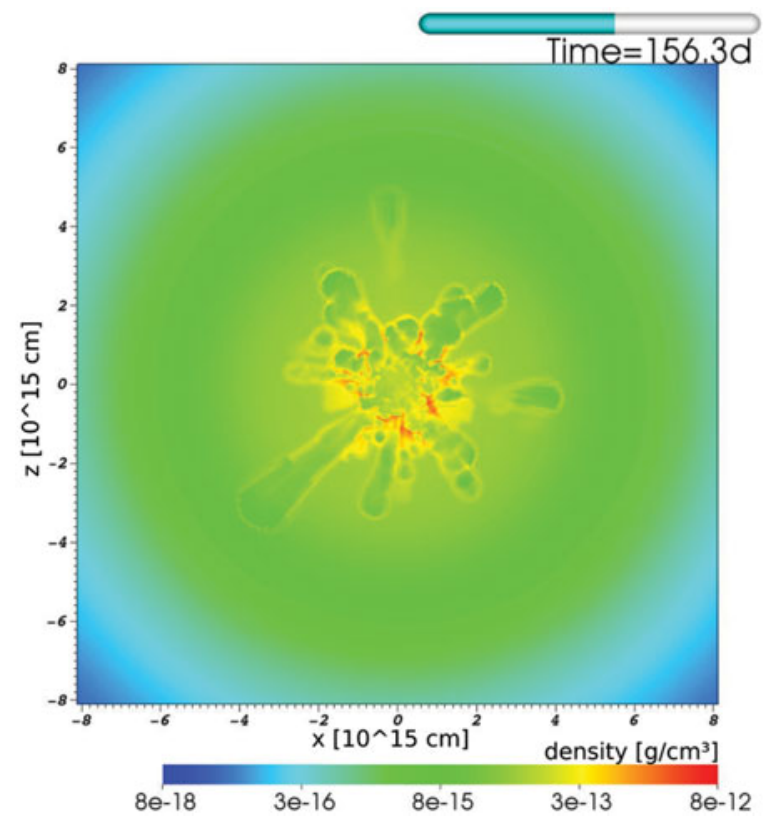

Figure 2. Crossectional slice through the young remnant for model B15, showing the mass density color coded. The walls of the Ni-rich domains have clearly higher densities than the interior of these structures.

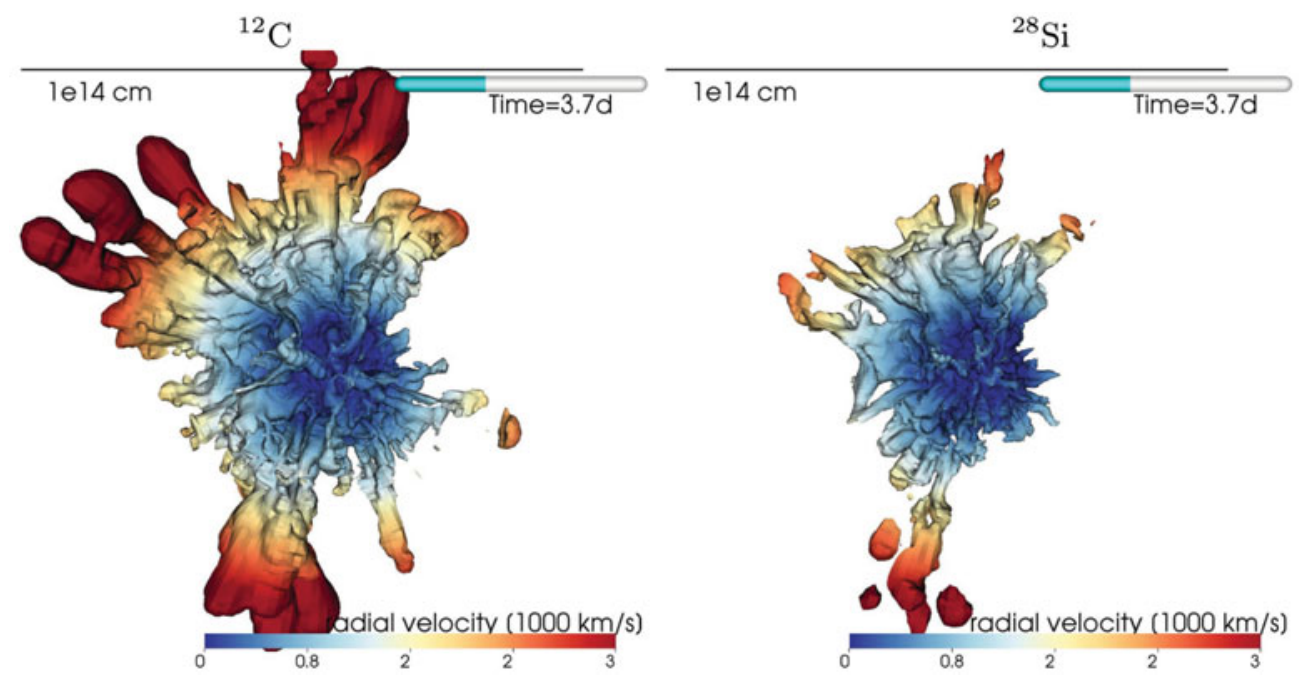

Figure 3. Surfaces enclosing $25 \%$ of ${ }^{12} \mathrm{C}$ (left) and ${ }^{28} \mathrm{Si}$ (right) with the highest respective densities for model W15.

This effect is displayed in Figure 2, where we plot a density slice of model B15 at around $150 \mathrm{~d}$. Clearly, there are density enhancements at the borders of the Ni-rich structures. One also can identify a central volume which is less dense than the walls of the bubbles. ('Nickel bubble', Woosley (1988))

Observations are not only limited to Fe, for which we show most of the plots. To show the differences to other elements we plot the surfaces containing each $25 \%$ of mass with the highest densities of ${ }^{12} \mathrm{C}$ and ${ }^{28} \mathrm{Si}$ in Figure 3 . As expected the heavier element ${ }^{28} \mathrm{Si}$ is 
located inside the volume where ${ }^{12} \mathrm{C}$ is found. Other elements may be distributed slightly differently. Similar differences arise for one element but different threshold densities. When comparing to observations, it is thus important to make sure that the structures seen and the ones in the models correspond to the same component of ejected material. For example, in the right panel of Figure 3 it looks like there is only one dominant finger, while in the left there are three.

\section{Acknowledgements}

This work was supported by the Deutsche Forschungsgemeinschaft through the Cluster of Excellence EXC 153 'Origin and Structure of the Universe' (www.universe-cluster.de), and by the EU through ERC-AdG No. 341157-COCO2CASA. Computer time at the Rechenzentrum Garching (RZG) and at the C2PAP-cluster at the Leibnitz-Rechenzentrum (LRZ) is acknowledged.

\section{References}

Arnett, D., Fryxell, B., \& Mueller, E. 1989, ApJ, 341, L63

Blondin, J. M. \& Lundqvist, P. 1993, ApJ, 405, 337

Blondin, J. M., Borkowski, K. J., \& Reynolds, S. P. 2001, ApJ, 557, 782

Boggs, S. E., Harrison, F. A., Miyasaka, H., et al. 2015, Science, 348, 670

Colella, P. \& Glaz, H. M. 1985, Journal of Computational Physics, 59, 264

Colella, P. \& Woodward, P. R. 1984, Journal of Computational Physics, 54, 174

Couch, S. M., Wheeler, J. C., \& Milosavljević, M. 2009, ApJ, 696, 953

Couch, S. M., Pooley, D., Wheeler, J. C., \& Milosavljević, M. 2011, ApJ, 727, 104

DeLaney, T., Rudnick, L., Stage, M. D., et al. 2010, ApJ, 725, 2038

Dewey, D., Dwarkadas, V. V., Haberl, F., Sturm, R., \& Canizares, C. R. 2012, ApJ, 752, 103

Ellinger, C. I., Young, P. A., Fryer, C. L., \& Rockefeller, G. 2012, ApJ, 755, 160

Ellinger, C. I., Rockefeller, G., Fryer, C. L., Young, P. A., \& Park, S. 2013, arXiv:1305.4137

Fesen, R. A. 2001, ApJS, 133, 161

Fesen, R. A., Hammell, M. C., Morse, J., et al. 2006, ApJ, 645, 283

Fryxell, B., Arnett, D., \& Mueller, E. 1991, ApJ, 367, 619

Gawryszczak, A., Guzman, J., Plewa, T., \& Kifonidis, K. 2010, A\& A, 521, A38

Grefenstette, B. W., Harrison, F. A., Boggs, S. E., et al. 2014, Nature, 506, 339

Grefenstette, B. W., Fryer, C. L., Harrison, F. A., et al. 2017, ApJ, 834, 19

Hachisu, I., Matsuda, T., Nomoto, K., \& Shigeyama, T. 1990, ApJ, 358, L57

Hachisu, I., Matsuda, T., Nomoto, K., \& Shigeyama, T. 1992, ApJ, 390, 230

Hachisu, I., Matsuda, T., Nomoto, K., \& Shigeyama, T. 1994, A\&AS, 104,

Hammer, N. J., Janka, H.-T., \& Müller, E. 2010, ApJ, 714, 1371

Herant, M. \& Benz, W. 1991, ApJ, 370, L81

Herant, M. \& Woosley, S. E. 1994, ApJ, 425, 814

Herant, M. \& Benz, W. 1992, ApJ, 387, 294

Hungerford, A. L., Fryer, C. L., \& Warren, M. S. 2003, ApJ, 594, 390

Hwang, U., Laming, J. M., Badenes, C., et al. 2004, ApJ, 615, L117

Hungerford, A. L., Fryer, C. L., \& Rockefeller, G. 2005, ApJ, 635, 487

Iwamoto, K., Young, T. R., Nakasato, N., et al. 1997, ApJ, 477, 865

Joggerst, C. C., Woosley, S. E., \& Heger, A. 2009, ApJ, 693, 1780

Joggerst, C. C., Almgren, A., Bell, J., et al. 2010, ApJ, 709, 11

Joggerst, C. C., Almgren, A., \& Woosley, S. E. 2010, ApJ, 723, 353

Junde, H., Su, H., \& Dong, Y. 2011, Nuclear Data Sheets, 112, 1513

Kageyama, A. \& Sato, T. 2004, Geochemistry, Geophysics, Geosystems, 5, Q09005

Kifonidis, K., Plewa, T., Janka, H.-T., \& Müller, E. 2000, ApJ, 531, L123

Kifonidis, K., Plewa, T., Janka, H.-T., \& Müller, E. 2003, A\&̈A, 408, 621 
Kifonidis, K., Plewa, T., Scheck, L., Janka, H.-T., \& Müller, E. 2006, A\&A, 453, 661

Li, H., McCray, R., \& Sunyaev, R. A. 1993, ApJ, 419, 824

Milisavljevic, D., \& Fesen, R. A. 2013, ApJ, 772, 134

Milisavljevic, D., \& Fesen, R. A. 2015, Science, 347, 526

Mueller, E., Fryxell, B., \& Arnett, D. 1991, A\&A, 251, 505

Nagataki, S., Hashimoto, M.-a., Sato, K., Yamada, S., \& Mochizuki, Y. S. 1998, ApJ, 492, L45

Ono, M., Nagataki, S., Ito, H., et al. 2013, ApJ, 773, 161

Orlando, S., Miceli, M., Pumo, M. L., \& Bocchino, F. 2015, ApJ, 810, 168

Orlando, S., Miceli, M., Pumo, M. L., \& Bocchino, F. 2016, ApJ, 822, 22

Plewa, T. \& Müller, E. 1999, A\&A, 342, 179

Podsiadlowski, P., Morris, T. S., \& Ivanova, N. 2007, Supernova 1987A: 20 Years After: Supernovae and Gamma-Ray Bursters, 937, 125

Potter, T. M., Staveley-Smith, L., Reville, B., et al. 2014, ApJ, 794, 174

Tanaka, T. \& Washimi, H. 2002, Science, 296, 321

Utrobin, V. P., Wongwathanarat, A., Janka, H.-T., \& Müller, E. 2015, Aछ̈A, 581, A40

Wongwathanarat, A., Janka, H.-T., \& Müller, E. 2010, ApJ, 725, L106

Wongwathanarat, A., Hammer, N. J., \& Müller, E. 2010, A\& A, 514, A48

Wongwathanarat, A., Janka, H.-T., \& Müller, E. 2013, A\&\&A, 552, A126

Wongwathanarat, A., Müller, E., \& Janka, H.-T. 2015, A\& A, 577, A48

Wongwathanarat, A., Janka, H.-T., Mueller, E., Pllumbi, E., \& Wanajo, S. 2016, arXiv: 1610.05643

Woosley, S. E. 1988, ApJ, 330, 218

Yamada, S. \& Sato, K. 1990, ApJ, 358, L9 